\title{
On the electronic configuration in Pu: spectroscopy and theory
}

J. G. Tobin, P. Soderlind, A. Landa, K. T. Moore, A. J. Schwartz, B. W. Chung, M. Wall, J. M. Wills, O. Eriksson, R. Haire, A. L. Kutepov

October 17, 2006

Journal of Physics: Condensed Matter 
This document was prepared as an account of work sponsored by an agency of the United States Government. Neither the United States Government nor the University of California nor any of their employees, makes any warranty, express or implied, or assumes any legal liability or responsibility for the accuracy, completeness, or usefulness of any information, apparatus, product, or process disclosed, or represents that its use would not infringe privately owned rights. Reference herein to any specific commercial product, process, or service by trade name, trademark, manufacturer, or otherwise, does not necessarily constitute or imply its endorsement, recommendation, or favoring by the United States Government or the University of California. The views and opinions of authors expressed herein do not necessarily state or reflect those of the United States Government or the University of California, and shall not be used for advertising or product endorsement purposes. 


\section{On the electronic configuration in Pu: spectroscopy and theory}

J.G. Tobin ${ }^{1}$, P. Söderlind ${ }^{1}$, A. Landa ${ }^{1}$, K.T. Moore ${ }^{1}$, A.J. Schwartz ${ }^{1}$, B. W. Chung ${ }^{1}$, M. Wall ${ }^{1}$, J.M. Wills ${ }^{2}$, O. Eriksson ${ }^{3}$, R. Haire ${ }^{4}$, and A.L. Kutepov ${ }^{5}$

${ }^{1}$ Lawrence Livermore National Laboratory, Livermore, CA 94550, USA

${ }^{2}$ Los Alamos National Laboratory, Los Alamos, NM 87545, USA

${ }^{3}$ Condensed Matter Theory Group, Physics Dept, Uppsala University, SE-75121 Uppsala, SWEDEN

${ }^{4}$ Oak Ridge National Laboratory, Oak Ridge, TN, USA

${ }^{5}$ Russian Federation Nuclear Center, Inst. of Technical Physics (VNIITF), Snezhinsk, Russia

Photoelectron spectroscopy, synchrotron-radiation-based x-ray absorption, electron energy-loss spectroscopy, and density-functional calculations within the mixed-level and magnetic models, together with canonical band theory have been used to study the electron configuration in $\mathrm{Pu}$. These methods suggest a $5 f^{n}$ configuration for $\mathrm{Pu}$ of $5 \leq n<6$, with $n \neq 6$, contrary to what has recently been suggested in several publications. We show that the $n=6$ picture is inconsistent with the usual interpretation of photoemission and x-ray absorption spectra. Instead, these spectra support the traditional conjecture of a $5 f^{5}$ configuration in $\mathrm{Pu}$ as is obtained by densityfunctional theory. We further argue, based on $5 f$-band filling, that an $n=6$ hypothesis is incompatible with the position of $\mathrm{Pu}$ in the actinide series and its monoclinic ground-state phase.

PACS numbers: 71.15.Mb; 71.20.-b; 71.10.-w, 71.20.Gj, 71.27.+a, 71.28.+d

*Contact Author

Tel: $\quad 925-422-7247$

Fax: $\quad 925-423-7040$

Email: tobin1@,1lnl.gov 


\section{On the electronic configuration in Pu: spectroscopy and theory}

The combined efforts of experiment and theory have promoted the understanding of the actinides considerably during recent years. For instance, diamond-anvil-cell studies of pressure-induced phase transitions in the actinides are well accounted for by density-functional theory (DFT) and explained by the broadening of $5 f$ bands that become less able to sustain the distorted geometries seen in especially the more $5 f$ dominated metals $\mathrm{U}, \mathrm{Np}$, and $\mathrm{Pu}$ [1]. Although phase instabilities are direct consequences of the narrow $5 f$-band electronic structure, the electronic and magnetic structures of plutonium, in particular, are still being debated [2-9] and not fully understood. It is important to clarify the situation because only from this understanding can a consistent picture of plutonium emerge. The unusual properties of $\mathrm{Pu}$ are well known and include, but are not limited to, a complex phase diagram with many high-symmetry and low-symmetry phases, large volume expansions associated with the phase transitions, and a combination of large positive $(\square, \square$, and $\square$ and negative ( $\square$ and $\square$ ') thermal expansion [10].

Recent experiments aimed at illuminating the electronic structure include photoemission [11], x-ray absorption and electron energy loss [12], magnetic susceptibility [13], and heat capacity [14]. Theoretically, DFT that allows for formation of spin and orbital moments $[6,15]$ appears to accurately determine total energies as well as some, but not all, aspects of the electronic structure of the known phases of $\mathrm{Pu}$, with the particular question of non-zero magnetic moments for $[-\mathrm{Pu}$. The mixed-level-model [2] is able to reproduce photoemission spectra of $\mathrm{Pu}$ in detail within a completely "non-magnetic" model, but has for now been restricted to the $\square$ phase and has the problem of artificially separating localized and delocalized $5 f$ states. Also, an approach based on the dynamical mean-field theory (DMFT) [4] has been applied to the $\square$ phase, although the interpretation of this work is unclear and has changed over time [5]. Until recently, the valence-electron configuration of $\mathrm{Pu}$ has not been questioned in either of the aforementioned experiments or models, but the around-mean-field (AMF) LSDA+U theory [8] suggests an 


\section{On the electronic configuration in Pu: spectroscopy and theory}

americium-like, non-magnetic $(\mathrm{J}=0) 5 f^{6}$ configuration for Pu. Independently, an LDA + U + SO (spin orbit) approach was pursued by Shorikov et al., [9] who arrived at the same conclusion. On its own, as also mentioned by the authors [8], this model does not reproduce the known photoemission spectra due to the formation of a pseudo-gap at the Fermi level, a recognized problem with the LSDA+U approach [3]. This deficiency was, however, addressed in an improved scheme that includes dynamical electron correlations (AMF-DMFT) [8].

In this paper we investigate the possibility of a $5 f^{6}$ configuration for Pu by means of photoemission, $\mathrm{x}$-ray absorption, electron energy loss spectroscopy and electronic-structure theory.

\section{X-ray Absorption and Electron Energy Loss Spectroscopy}

The results from $\mathrm{x}$-ray absorption and electron energy loss spectroscopy indicate that the number of $5 f$ electrons, $n$, of Pu must be at least as great as 5 and less than 6 . The equivalence of the XAS and high-energy EELS measurements has already been demonstrated for $\mathrm{Ce}$ and $\mathrm{Pu}$, as well as other actinides. [12, 16-19] Now, the argument against $n=6$ will be discussed in a stepwise fashion.

\section{The relative diminishment of the $\mathrm{Pu} 4 d_{3 / 2}$ peaks indicates strong relativistic effects in the Pu $5 f$ states, i.e. a jj-coupling or jj-skewed intermediate case coupling scheme.} As can be seen in Figure 1, the intensity of the $4 d_{3 / 2}$ peak of Pu is significantly reduced versus that of $U$ [20]. This large reduction is driven by the electric dipole selection rule that forbids transition from a pure $d_{3 / 2}$ peak into a pure $f_{7 / 2}$ peak. It also implies that the Pu $5 f$ states must be split into two lobes, the lower (mainly occupied) lobe being principally of pure $5 / 2$ character and the upper (unoccupied) lobe being of principally pure 7/2 character. This picture is shown schematically in Figure 2. This result is independent of any particular computational model of 


\section{On the electronic configuration in Pu: spectroscopy and theory}

schemes for spin-orbit splitting or the calculation of x-ray absorption cross section and thus does not depend upon the details of the branching ratio analysis presented previously. $[12,18]$

\section{Coupled with the result in I above, the absence of a pre-peak in the Pu $5 d \mathrm{XAS}$ and} EELS indicates that $\boldsymbol{n}$ must be at least as great as 5.

As shown in Figure 3, there is a pre-peak in the EELS $5 d$ to $5 f$ transition for Th and U, but not for Pu. Similar results (Figure 4) have been obtained for 5d XAS. [16] The pre-peak structure in the $4 d$ XAS of the rare earths was explained many years ago by Dehmer et al. [21] : it is driven by the combination of angular momentum coupling between the $4 d$ and $4 f$ states and the dependence of the Coulomb energy term upon the details of this coupling. The spectroscopic transition for the rare earths can be summarized as follows.

$4 d^{10} 4 f^{n}+\mathrm{h} \square \rightarrow 4 d^{9} 4 f^{n+1}$

Eq A

When $n=14$, there is no transition. When $n=13$, there is a single main peak, without any prepeaks. [16] This is because partial occupation of both the $d$ and $f$ states is required for coupling. If $n=13$ and $n+1=14$, then in the final state the $4 f$ level is filled and no coupling occurs. For the lighter actinides $(n<6)$, the situation is similar but not identical.

$5 d_{5 / 2}$, pure jj, $n<6$

Eq B1

$\left(5 d_{3 / 2}\right)^{4}\left(5 d_{5 / 2}\right)^{6}\left(5 f_{5 / 2}\right)^{n}\left(5 f_{7 / 2}\right)^{0}+\mathrm{hv} \rightarrow\left(5 d_{3 / 2}\right)^{4}\left(5 d_{5 / 2}\right)^{5}\left(5 f_{5 / 2}\right)^{n+1}\left(5 f_{7 / 2}\right)^{0}+\left(5 d_{3 / 2}\right)^{4}\left(5 d_{5 / 2}\right)^{5}\left(5 f_{5 / 2}\right)^{\mathrm{n}}\left(5 f_{7 / 2}\right)^{1}$

$5 d_{3 / 2}$, pure $\mathrm{jj}, n<6$

Eq B2

$\left(5 d_{3 / 2}\right)^{4}\left(5 d_{5 / 2}\right)^{6}\left(5 f_{5 / 2}\right)^{\mathrm{n}}\left(5 f_{7 / 2}\right)^{0}+\mathrm{hv} \rightarrow\left(5 d_{3 / 2}\right)^{3}\left(5 d_{5 / 2}\right)^{6}\left(5 f_{5 / 2}\right)^{n+1}\left(5 f_{7 / 2}\right)^{0}$ 


\section{On the electronic configuration in Pu: spectroscopy and theory}

Again transitions from $d_{3 / 2}$ to $f_{7 / 2}$ are forbidden. For the pre-peaks at lower $\mathrm{h} \square$, we need only worry about the $\left(5 f_{5 / 2}\right)^{n+1}$ terms. (Transitions into the $f_{7 / 2}$ states will generally need higher energies and pre-peaks that might be associated with this transition will be coincident with the main $f_{5 / 2}$ spectral structure and lost therein.) The final state $5 / 2$ level will be filled at $n+1=6$ or $n=5$. For $n<5$ (e.g., Th and $\mathrm{U}$ ), there will be pre-peaks. The absence of pre-peaks for Pu, both $\square$, and $\square$, indicates that $n$ must be 5 or greater. This raises the question: what about $n$ of 6 and above? For $n \geq 6$, the transition looks like this.

$5 d_{5 / 2}$, pure jj, $n \geq 6$ Eq C1

$\left(5 d_{3 / 2}\right)^{4}\left(5 d_{5 / 2}\right)^{6}\left(5 f_{5 / 2}\right)^{6}\left(5 f_{7 / 2}\right)^{\mathrm{n}-6}+\mathrm{hv} \rightarrow\left(5 d_{3 / 2}\right)^{4}\left(5 d_{5 / 2}\right)^{5}\left(5 f_{5 / 2}\right)^{6}\left(5 f_{7 / 2}\right)^{\mathrm{n}-5}$

$5 d_{3 / 2}$, pure $\mathrm{jj}, \mathrm{n} \geq 6$

Eq C2

$\left(5 d_{3 / 2}\right)^{4}\left(5 d_{5 / 2}\right)^{6}\left(5 f_{5 / 2}\right)^{6}\left(5 f_{7 / 2}\right)^{\mathrm{n}-6}+\mathrm{hv} \rightarrow$

No Transition Allowed

Once again, there will be no transition in Eq C2 because $d_{3 / 2}$ to $f_{7 / 2}$ transitions are forbidden.

The situation is different in another, subtle, way as well. A filled $5 f_{5 / 2}$ level now stands between the partially occupied $5 d$ and $5 f_{7 / 2}$ levels. It is unclear whether angular momentum coupling and thus pre-peak formation will occur under these conditions. In fact, the spectrum from $\mathrm{AmH}_{2}$ shown in Figure 3 exhibits no evidence of any pre-peaks. Although this is not a spectrum from an elemental actinide, past experiments have shown that for a given actinide, the peak ratio values tend to be grouped together, with larger separations between the elemental groups. [22] The grouping tended to be tightest for actinide elements that exhibited a strong localization even in the elemental, metallic state. 


\section{On the electronic configuration in Pu: spectroscopy and theory}

\section{III.The presence of two edges in the Pu $5 d$ XAS and a substantial peak in the $4 d_{3 / 2}$ XAS indicate that $n<6$.}

As can be seen in Figure 4, in the $5 d$ XAS of both $\square$ - and $\square-\mathrm{Pu}$, there are two strong edges in the vicinity of $\mathrm{h} \square=110$ to $120 \mathrm{eV}$. Similar, albeit weaker, structure is observed in the EELS of $\square$ and $\square-\mathrm{Pu}$, as shown in Figure 3. These two steps are the leading edges of the main $5 d_{5 / 2}$ (about $110 \mathrm{eV}$ ) and $5 d_{3 / 2}$ (about $120 \mathrm{eV}$ ) transitions. (Confirmation of the initiation of the $5 d$ transition near $110 \mathrm{eV}$ can be gleaned from photoelectron spectroscopy. See Ref. 23 and references therein.) As illustrated in $\mathrm{Eq} \mathrm{C} 2$, if $n=6$ or more, one of the two transitions is lost. This is exactly what has happened in $\mathrm{AmH}_{2}$, as shown in Figure 3. Here, the second spin-orbit split peak is essentially gone or at least strongly attenuated and lost in the tail of the other peak/edge. The overall peak structure of the "giant resonance" of $\mathrm{AmH}_{2}$ is now about half as wide as that of the $\mathrm{Pu}$. Thus, the presence of two strong edges indicates that $n<6$ for $\mathrm{Pu}$.

An analogous process will occur for the $4 d$ to $5 f$ transitions. For a pure jj coupling scheme and electric dipole selection rules, the $4 d_{3 / 2}$ peak should vanish. In reality, there will be mixing between the pure $5 f_{5 / 2}$ and pure $5 f_{7 / 2}$ states, so at $n=6$, one would expect a small but almost negligible $4 d_{3 / 2}$ peak. Again, this is exactly what has been observed for $\mathrm{AmH}_{2}$ as shown in Figure 5. [22] The $\mathrm{Pu} 4 d_{3 / 2}$ peak is too large for $n=6$. Thus, for $\mathrm{Pu}, n<6$.

What about the possibilities for Am being $n>6$ ? This seems unlikely. As will be discussed below, there is very strong evidence that Am has $n=6$. Additionally, recent measurements on $\mathrm{Cm}$ [24] indicate that $n=7$ for Cm, within an LS like coupling scheme. The return to an LS skewed intermediate case for the heavier actinides is not inconsistent with the atomic model of 


\section{On the electronic configuration in Pu: spectroscopy and theory}

van der Laan and Thole [25], which has been used to explain the cross-sectional behavior of the lighter actinides. $[12,18]$

Before going onto a discussion of photoelectron spectroscopy, it is useful to consider the possibility of phase specific variations of the XAS/EELS and thus the $n$ value for Pu. As can be seen in Figures 3 and 4, within each technique, the results for $\square$ and $\square$ are quite similar for the $5 d$ to $5 f$ transitions. Additionally, as shown in Figure 6, the $N 4,5$ electron energy loss spectra for $\square$ and $\square \mathrm{Pu}$ are again rather similar. [22] The experimental data hence point towards the generality of the conclusion that for $\mathrm{Pu} n$ is less than 6 and greater than or equal to 5, whether for the $\square$ or the $\square$ phase. This does not rule out that there may be phase specific variations between the spectra, but these are small compared to the variation from element to element.

Thus, to summarize for Pu $5 f$ occupation: from XAS and EELS, $5 \leq n<6$, with $n \neq 6$.

\section{Valence Band Photoelectron Spectroscopy (PES)}

Photoelectron spectroscopy measures transitions between states, in which an electron is ejected from the material. If the measurements are angle resolved and the samples single crystalline, then one may glean information about the dispersion of specific states from the PES of the valence bands. If the sample is polycrystalline or a significant angle averaging is applied, then one may get information about the initial density of states, as in ESCA or the electron spectroscopy for chemical analysis, usually performed at fairly high photon energies. In the case of $\mathrm{Pu}$, invariably the samples are not single-crystalline and most of the high-energy resolution valence band PES has been performed at low energies using HeI $(21.22 \mathrm{eV})$ or He II $(40.8 \mathrm{eV})$

excitation sources. $[11,26]$ For $\mathrm{Pu}$, there has also been a history of interpretation of the spectral features in terms of multiplet structures. [27,28] In the case of the ESCA of Pu with laboratory
LLNL
Page 7
10/10/06 


\section{On the electronic configuration in Pu: spectroscopy and theory}

sources (e.g. with $\mathrm{MgK}-\square$ at $\mathrm{h} \square=1254 \mathrm{eV}$ ) the resolution is not sufficient to resolve the fine structure of interest in the valence bands. [29] However, there is one synchrotron-radiation-based study of $\mathrm{Pu}$, which can provide significant guidance concerning how to interpret the valence band PES of $\mathrm{Pu}[23]$.

1. Other than intensity variations and a slight loss of fine structure with increasing energy, the valence band spectra of $\mathrm{Pu}$ are the same over a very wide photon energy range. This result is shown in Figure 7. The photon energies include $\mathrm{h} \square=225 \mathrm{eV}$ (at the Cooper minimum), 180 $\mathrm{eV}$ (off-resonance), $125 \mathrm{eV}$ (on resonance) and $100 \mathrm{eV}$ (at the anti resonance). [23] In all of these, the spectral structure is essentially the same: a peak near the Fermi Level, followed by a minimum at a binding energy of $1 / 2 \mathrm{eV}$ and a broad maximum at about $1 \mathrm{eV}$.

Qualitatively, this series of spectra suggests some underlying constancy to the PES response of $\square \mathrm{Pu}$ and validates the previous lower energy studies done using He I and He II laboratory sources.

2. There is no observable multiplet structure in the $\mathrm{Pu} 6 p_{1 / 2}$ spectra. This can be seen in Figure 8. Unlike the $\mathrm{Ce} 5 p_{1 / 2}$ spectrum, the $\mathrm{Pu} 6 p_{1 / 2}$ spectrum shows no multiplet structure. The $\mathrm{Pu}$ $6 p$ feature is wider due to the increased lifetime broadening of the actinides relative to the rare earths, but considering the size and energy separation of the Ce satellite peaks, if there was comparable structure in the $\mathrm{Pu} 6 p_{1 / 2}$ spectrum, it should be observable. In the case of Ce, multiplet structure is observable in both the valence bands [30] and the shallow core level $\left(5 p_{1 / 2}\right)$. The absence of multiplet structure in the $\mathrm{Pu} 6 p_{1 / 2}$ spectrum strongly calls into question the process of analyzing the valence bands of $\mathrm{Pu}$ within the usual multiplet structure approach [27,28]. 


\section{On the electronic configuration in Pu: spectroscopy and theory}

These two results taken together strongly suggest the following: The valence band spectra of 마-Pu are representative of the electron density of states and can be compared directly to the predictions of electronic-structure theory.

Now when we have confidently established that photoemission spectra (PES) in fact correspond to electronic density-of-states (DOS), we can immediately compare our spectra with theory to help distinguish between some of the proposed models. In Fig. 9 we show our measured PES together with calculations of convoluted DOS [31] by Eriksson et al. [2], Söderlind et al. [6], Pourovskii et al. [8] and Kutepov and Kutepova [32]. The details of these calculations can be found in Refs. 2, 6, 8, and 32. The first of these models is referred to as the mixed-level-model (MLM) and assumes a $5 f^{5}$ configuration for $\mathrm{-}-\mathrm{Pu}$, that is divided into electrons with localized (4) and itinerant character (1). The MLM does not allow formation of magnetic moments, whereas the second model, spin/orbital polarized DFT (POL-DFT) does, but still predicts a $5 f^{5} \mathrm{Pu}$ valence. This latter calculation is in many respects very similar to that of Kutepov and Kutepova [32] whose results confirmed $n \sim 5$. In contrast to these approaches, the more recent models by Pourovskii et al. [8] and Shorikov et al. [9], dictate a non-magnetic $5 f^{6}$ configuration of Pu.

Notice in Fig. 9 that the $5 f^{5}$-configuration models (MLM and POL-DFT) both capture the very narrow peak close to the Fermi level in close agreement to the measured spectra, but in complete disagreement with the AMF-DMFT result. [8] Also, the broad feature at $\sim-0.25 \mathrm{eV}$ in the AMF-DMFT does not correspond to PES, which instead shows a shallow minimum close to $0.2 \mathrm{eV}$. Contrary to the AMF-DMFT model, the MLM and DFT-POL compares well with PES throughout the entire energy range. Hence, PES does not support the conjecture of a $\mathrm{Pu} 5 f^{6}$ configuration but rather the more traditional view of $5 f^{5}$. 


\section{On the electronic configuration in Pu: spectroscopy and theory}

\section{5 f occupation and crystal structure}

The previous sections provide strong experimental support for the $5 f^{5}$ picture of $\mathrm{Pu}$. Here we will show that this is entirely consistent with the view of Pu being part of the actinide series of metals. First we need to point out that DFT has been a remarkably successful tool in not only understanding ground-state properties of the actinides but also predict some. Generally, Th is considered being the first actinide metal because in Ac no $f$ states are occupied and Th is the first metal to have an appreciable amount of $5 f$ electrons in the periodic table of elements. Even though for Th this fraction is rather small ( 0.5) Johansson et al. [33] showed that it is responsible for the ground-state face-centered cubic (fcc) structure. Eriksson et al. [34] determined that due to the promotion of $5 f$ electrons (mainly from the $6 d$ electrons) under compression, Th undergoes a phase transition to a body-centered tetragonal phase similar to the ground-state phase of the proceeding metal, Pa. DFT calculations are actually able to very accurately reproduce the measured pressure-behavior of the tetragonal distortion of $\mathrm{Ce}$ and $\mathrm{Th}$ which has a known linkage to the filling of the $f$-band [35]. Söderlind and Eriksson [36] studied the crystal structure of $\mathrm{Pa}$ and predicted a phase transition to a high-pressure face-centered orthorhombic phase very close to that of $\square-\mathrm{U}$. This was understood to be a consequence of a subtle pressure-induced increase of the $5 f$-band occupation in $\mathrm{Pa}$, and the orthorhombic phase was later confirmed by diamond-anvil-cell experiments [37]. The detailed study of uranium by Akella et al. [38] showed a very similar result, with DFT accurately reproducing both the ground-state crystal structure as well as its delicate changes with pressure. In fact, the groundstate phase of all the early actinides (Th-Pu) is correctly determined by careful DFT computations [1].

Clearly, the population of the $5 f$ states is of uttermost importance for the ground-state properties, including the crystal structure, and DFT provides a unified picture of this relationship
LLNL
Page 10
$10 / 10 / 06$ 


\section{On the electronic configuration in Pu: spectroscopy and theory}

for the actinides as described above. In Fig. 10 we plot the DFT predictions of $6 d$ and $5 f$ occupations for Th-Cm, together with the AMF-DMFT result for Pu. Notice that the DFT occupation numbers vary smoothly over the series whereas the Pu AMF-DMFT [8] (5f $f^{6}$ configuration implies a rather substantial kink going from $\mathrm{Np}$ to $\mathrm{Pu}$, which appears to be inconsistent with the overall trend. If this is correct, either $\mathrm{Pu}$ is not a natural member of the actinide series (the occupation numbers should vary smoothly as in the $d$-transition metals or the rare-earth series) or the DFT occupation numbers of Th-Np are all in error. None of these alternatives are very attractive. Notice also that DFT predicts Am and $\mathrm{Cm}$ to have 6.3 and $7.25 f$ electrons, respectively. This is consistent with a close to $5 f^{6}$ configuration in Am as discussed in previous section. Recent high-pressure diamond-anvil-cell experiments for Am and Cm [24] reveal several phase transitions under compression. Am and $\mathrm{Cm}$ behave relatively similarly under pressure with the striking exception of a monoclinic $(\mathrm{C} 2 / \mathrm{c})$ phase in $\mathrm{Cm}(\mathrm{CmIII})$. DFT calculations $[24,38-40]$ reproduce these phase transitions very accurately including the distinction of a monoclinic phase in $\mathrm{Cm}$. This gives credence to the predicted occupation numbers because the structural behavior depends sensitively on this property. Let us next investigate this in detail using a simple but transparent model.

The crystal-structure dependence on the 5f-band filling was previously investigated [42] by the so-called canonical band theory when electrostatic interactions and Born-Mayer repulsion were accounted for. Within this theory, one can estimate the energy of any geometrical configuration of atoms as a function of $5 f$-band population, in analogy to what was done previously to understand the crystal structures of the non-magnetic $d$-transition [43] and rareearth [44] metals. Canonical band theory shows that for $5 f^{3}, 5 f^{4}$, and $5 f^{5}$ configurations, the $\square-\mathrm{U}$,

५-Np, and $\mathrm{C-Pu}$ structures are favored, hence suggesting that $\mathrm{U}, \mathrm{Np}$, and $\mathrm{Pu}$ have about 3,4 , and $55 f$ electrons, respectively. In the present study we again apply this canonical model with a
LLNL 


\section{On the electronic configuration in Pu: spectroscopy and theory}

special interest in the occupation of $65 f$ electrons. In Fig. 11 we show the structural stability of several phases for $f$ occupations between 0 and 14 . Here we are interested in crystal structures that are stabilized $5 f$ bonding, and leave out the Am ground-state double-hexagonal phase, which is known to be a consequence of $6 d$ bonding. Notice that for $6 f$ electrons, a primitive orthorhombic phase (p.o.: pnma with $b / a \sim 0.8, c / a \sim 0.7, x \sim 0.4$, and $z \sim 0.1$ ) is stable over the $\square$ $\mathrm{Pu}$ phase. This suggests that if the $5 f^{6}$ configuration in $\mathrm{Pu}$ is correct, the ground-state phase would not be $\square-\mathrm{Pu}$ but rather a primitive orthorhombic (p.o.) or some other unknown phase. Instead, this result tells us that for full $5 f$-electron bonding of 6 electrons, a p.o. phase is predicted. Assuming that $\mathrm{Am}$ (and not $\mathrm{Pu}$ ) has six $5 f$ electrons, this corresponds to Am with completely delocalized $5 f$ electrons. At ambient pressure Am is a "localized" metal with essentially non-bonding $5 f$ electrons. During compression, however, the $5 f$ orbitals will overlap and at sufficiently high pressure, they will "delocalize", i.e., participate and even dominate the chemical bonds. Consequently, the canonical-band picture predicts a p.o phase in Am at high pressure. This has indeed been established recently in diamond-anvil-cell work at $16 \mathrm{GPa}$ [45].

\section{Conclusions}

Traditionally, $\mathrm{Pu}$ is thought of as having $\sim 5 f$ electrons, which is consistent with DFT calculations and the general trend of the occupation numbers and crystal structures of the actinides. Recently, however, a distinct view has been proposed in several publications $[8,9,46]$ aimed at explaining the lack of credible evidence of large magnetic moments in $\mathrm{Pu}$. The idea is

founded on a $5 f^{6}$, americium-like, configuration in which the $5 f_{5 / 2}$ sub band is fully occupied and hence quench any possibility for magnetism in $\mathrm{Pu}$. We have shown, by two independent measurements (photoemission and x-ray absorption), that this is probably incorrect. We have further argued that a $5 f^{6}$ configuration in $\mathrm{Pu}$ is misleading because it is incompatible with its
LLNL
Page 12
$10 / 10 / 06$ 


\section{On the electronic configuration in Pu: spectroscopy and theory}

position in the series of actinides and its ground-state monoclinic phase. Evidently, all present theoretical approaches have failings and limitations. Additional improvements are necessary before it can be stated that the physics of Pu is fully understood. However, as we hopefully have convinced you, it is neither sufficient nor correct to force a non-magnetic " $n=6$ solution" upon the longstanding problem of the Pu $5 f$ electronic structure.

\section{Acknowledgements}

We thank Dr. L.V. Pourovskii for providing us with the AMF-DMFT DOS. This work was performed under the auspices of U.S. Department of Energy by the University of California, Lawrence Livermore National Laboratory under contract No. W-7405-Eng-48. 


\section{On the electronic configuration in Pu: spectroscopy and theory}

\section{References}

[1] P. Söderlind, O. Eriksson, B. Johansson, J.M. Wills, and A.M. Boring, Nature (London) 374, 524 (1995); P. Söderlind, Adv. Phys. 47, 959 (1998).

[2] O. Eriksson, J.D. Becker, A.V. Balatsky, and J.M. Wills, J. Alloys Compd. 287, 1 (1999).

[3] J. Bouchet, B. Siberchicot, F. Jollet, and A. Pasturel, J. Phys. Condens. Matter 12, 1723 (2000).

[4] S.Y. Savrasov, G. Kotliar, and E. Abrahams, Nature (London) 410, 793 (2001).

[5] A.C. Lawson, J.A. Roberts, B. Martinez, M. Ramos, G. Kotliar, F.W. Trouw, M.R. Fitzsimmons, M.P. Hehlen, J.C. Lashley, H. Ledbetter, R.J. McQueeny, A. Migliori, Phil. Mag. 86, 2713 (2006).

[6] P. Söderlind, EuroPhys. Lett. 55, 525 (2001); P. Söderlind, A. Landa, and B. Sadigh, Phys. Rev. B 66, 205109 (2002); P. Söderlind, J. Alloys Compd. (submitted).

[7] J.C. Lashley, A. Lawson, R.J. McQueeney, and G.H. Lander, Phys. Rev. B 72, 054416 (2005).

[8] A.B. Shick, V. Drchal, and L. Havela, EuroPhys. Lett. 69, 588 (2005); L.V. Pourovskii, M.I. Katsnelson, A.I. Lichtenstein, L. Havela, T. Gouder, F. Wastin, A.B. Shick, V. Drchal, and G.H. Lander, EuroPhys. Lett. 74, 479 (2006).

[9] A.O. Shorikov, A.V. Lukoyanov, M.A. Korotin, and V.I. Anisimov, Phys. Rev. B 72, 024458 (2005); arXiv:cond-mat/0412724 v2 21 Jan 2005.

[10] Plutonium Handbook A Guide to the Technology (Gordon and Breach, New York, 1967) ed. O.J. Wick.

[11] A.J. Arko, J.J. Joyce, L. Morales, J. Wills, J. Lashley, F. Wastin, and J. Rebizant, Phys. Rev. B 62, 1773 (2000).

[12] J.G. Tobin, K.T. Moore, B.W. Chung, M.A. Wall, A.J. Schwartz, G. van der Laan, and A.L. Kutepov, Phys. Rev. B 72, 085109 (2005). 


\section{On the electronic configuration in Pu: spectroscopy and theory}

[13] S. Meot-Raymond and J.-M, Fournier, J. Alloys Compd. 232, 119 (1996).

[14] P. Javorsky, L. Havela, F. Wastin, E. Colineau, and D. Bouexiere, Phys. Rev. Lett. 96, 156404 (2006).

[15] P. Söderlind and B. Sadigh, Phys. Rev. Lett. 92, 185702 (2004).

[16] K.T. Moore, M.A. Wall, A.J. Schwartz, B.W. Chung, D.K. Shuh, R.K. Schulze, and J.G. Tobin, Phys. Rev. Lett. 90, 196404 (May 2003) and references therein.

[17] K.T. Moore, B.W. Chung, S.A. Morton, S. Lazar, F.D. Tichelaar, H.W. Zandbergen, P. Söderlind, G. van der Laan, A.J. Schwartz, and J.G. Tobin, Phys. Rev. B 69, 193104 ( 2004).

[18] van der Laan, K.T. Moore, J.G. Tobin, B.W. Chung, M.A. Wall, and A.J. Schwartz, Phys. Rev. Lett. 93, 097401 (Aug 2004).

[19] K.T. Moore et al., Phil Mag 84(10) p1039

[20] J.G. Tobin et al, MRS Symp. Proc. 313, 619 (1993).

[21] J. L. Dehmer et al, Phys. Rev. Lett 26, 1521 (1971).

[22] K.T. Moore et al., PRB 73, 033109 (2006).

[23] J.G. Tobin et al., Phys. Rev. B 68, 155109 (2003).

[24] Heathman S, Haire RG, Le Bihan T, Lindbaum A, Idiri M, Normile P, Li S, Ahuja R, Johansson B, Lander G, SCIENCE 309 (5731): 110-113 JUL 1 2005; S. Heathman et al., Phys. Rev. Lett. 85, 2961 (2000).

[25] G. van der Laan and B. T. Thole, Phys. Rev. B 53, 14458 (1996).

[26] L. Havela et al, Phys. Rev. B 65, 235118 (2003).

[27] T. Gouder, R. Eloirdi, F. Wastin, E. Colineau, J. Rebizant, D. Kolberg, and F. Huber, Phys. Rev. B 70, 235108 (2004).

[28] J. R. Naegele, L. Manes, J. C. Spirlet, and W. Müller, Phys. Rev. Lett. 52, 1834-1837 (1984).

[29] R. Baptist et al, J. Phys. F (Metal Phys) 12, 2103 (1982).

[30] J.G. Tobin et al, Physica B 378-380, 925 (2006) and references therein.

$\begin{array}{lll}\text { LLNL } & \text { Page } 15 & 10 / 10 / 06\end{array}$ 


\section{On the electronic configuration in Pu: spectroscopy and theory}

[31] DOS convoluted as described in Ref. 11.

[32] A.L. Kutepov and I. Kutepova, J. Phys. Cond. Matter 15, 2607 (2003).

[33] B. Johansson, R. Ahuja, O. Eriksson, and J.M. Wills, Phys. Rev. Lett. 75, 3968 (1995).

[34] O. Eriksson, P. Söderlind, and J.M. Wills, Phys. Rev. B 45, 12588 (1992).

[35] P. Söderlind, O. Eriksson, B. Johansson, and J.M. Wills, Phys. Rev. B 52, 13169 (1995).

[36] P. Söderlind and O. Eriksson, Phys. Rev. B 56, 10719 (1997).

[37] R.G. Haire, S. Heathman, M. Iridi, T. Le Bihan, A. Lindbaum, and J. Rebizant, Phys. Rev. B 67, 134101 (2003).

[38] J. Akella, S. Weir, J.M. Wills, and P. Söderlind, J. Phys. Condens. Matter 9, L549 (1997).

[39] M. Penicaud, J. Phys. Condens. Mat. 143575 (2002).

[40] P. Söderlind and A. Landa, Phys. Rev. B 72024109 (2005).

[41] P. Söderlind, Mater. Res. Soc. Symp. Proc. 89315 (2006).

[42] P. Söderlind, J.M. Wills, and O. Eriksson, Phys. Rev. B 57, 1320 (1998).

[43] J.C. Duthie and D.G. Pettifor, Phys. Rev. Lett. 38, 564 (1977); H.L. Skriver, Phys. Rev. B 31, 1909 (1985).

[44] A.K. McMahan, H.L. Skriver, and B. Johansson, Phys. Rev. B 23, 5016 (1981).

[45] A. Lindbaum, S. Heathman, K. Litfin, Y. Meresse, R.G. Haire, T. Le Bihan, and H. Libotte, Phys. Rev. B 63, 214101 (2001).

[46] A. Shick, L. Havela, J. Kolorenc, V. Drchal, T. Gouder, and P.M. Oppeneer, Phys. Rev. B 73, 104415 (2006). 
On the electronic configuration in Pu: spectroscopy and theory

\section{4d $\rightarrow$ 5f XAS Transition \\ with $\mathrm{h} \square \approx 800 \mathrm{eV}$}

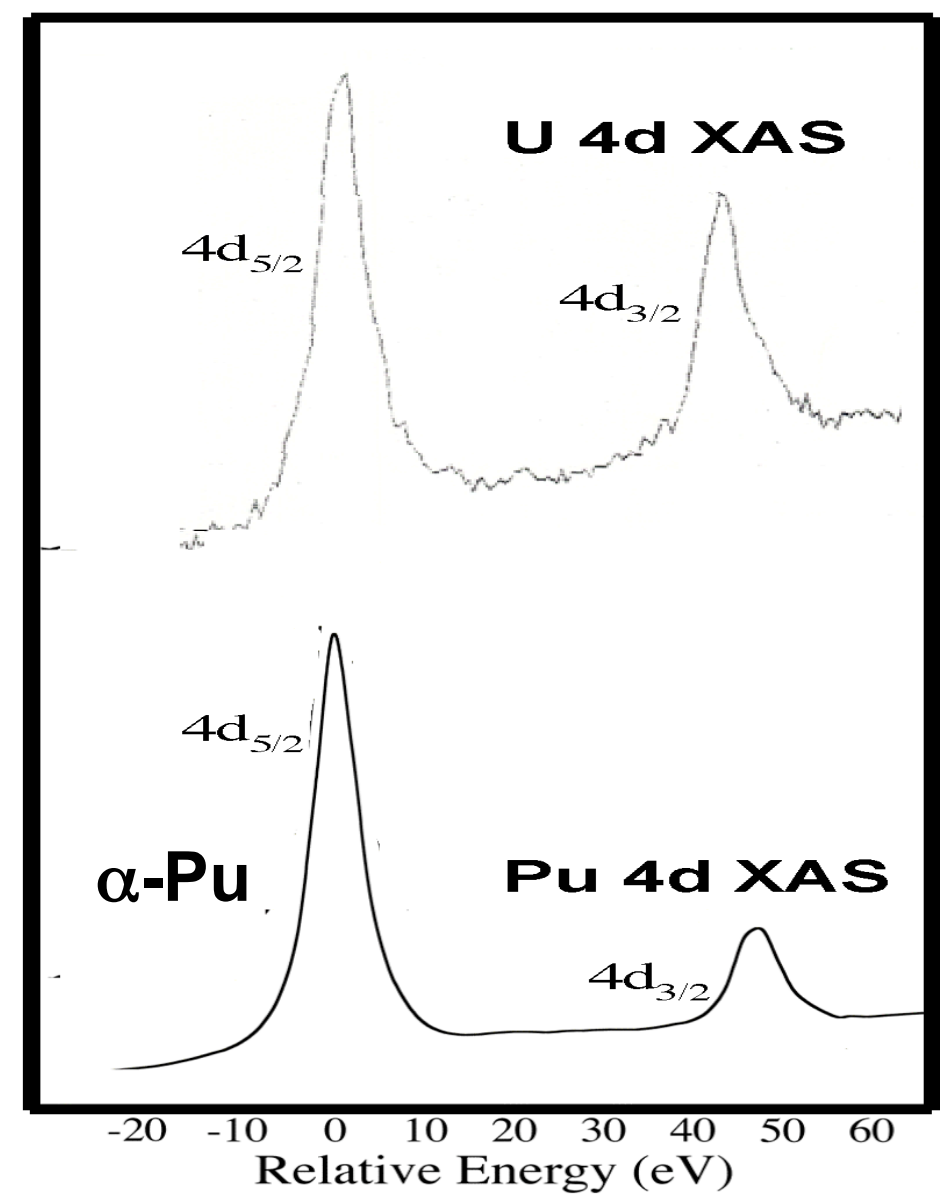

Figure 1

The XAS results for $\mathrm{Pu}$ [12] and $\mathrm{U}$ [20] are shown here. 
On the electronic configuration in Pu: spectroscopy and theory

\section{Simple Picture derived from the spectroscopic analysis}
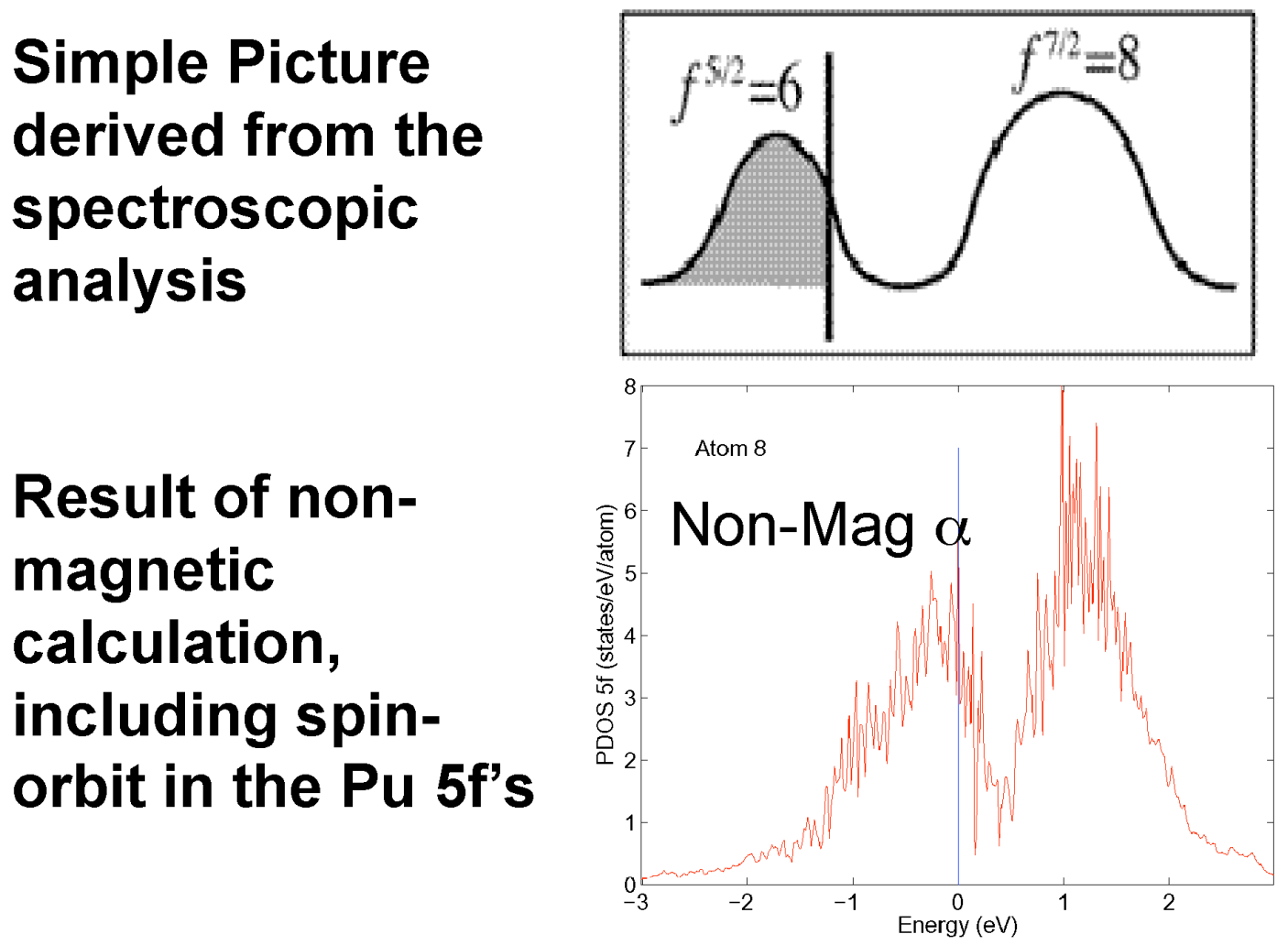

\section{Result of anti- ferromagnetic calculation, including spin- orbit in the Pu 5f's}

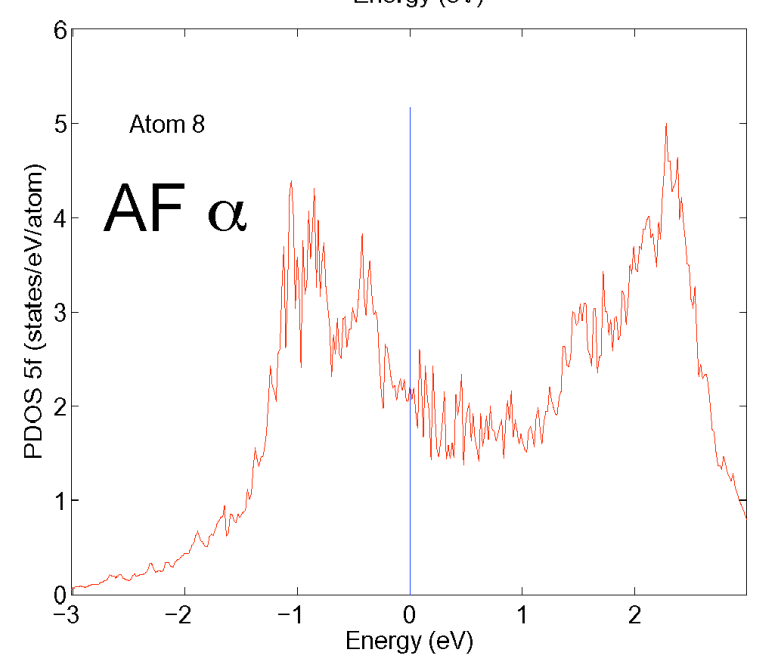

Figure 2

The two lobed structure of the Pu $5 f$ density of states is shown here. Top: The simple picture derived from the spectroscopic analysis. Middle: the result of non-magnetic calculation by Kutepov, including the spin-orbit splitting in the Pu $5 f$ states. Bottom: the result of an antiferromagnetic calculation by Kutepov, including the spin-orbit splitting in the Pu $5 f$ states. See Ref. 12 for the details concerning this figure. 


\section{On the electronic configuration in Pu: spectroscopy and theory}

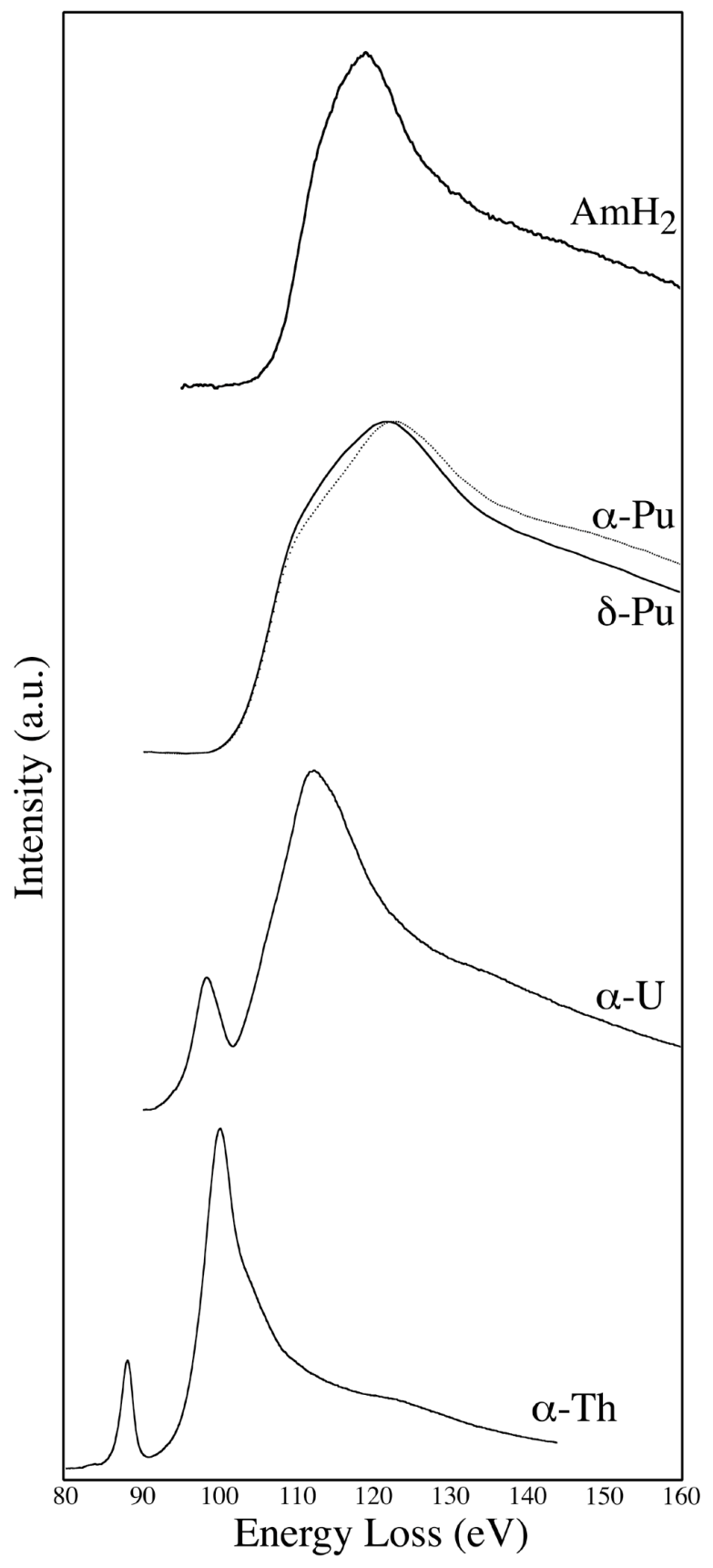

Figure 3

The EELS spectra of the $5 d$ to $5 f$ transitions of Th (bottom), U, (second from bottom), $\mathrm{Pu}$ (second from top) and $\mathrm{AmH}_{2}$ (top) are shown here. The Th, U and Pu data are from Ref 16. The $\mathrm{AmH}_{2}$ data are from Ref. 22. 
On the electronic configuration in Pu: spectroscopy and theory

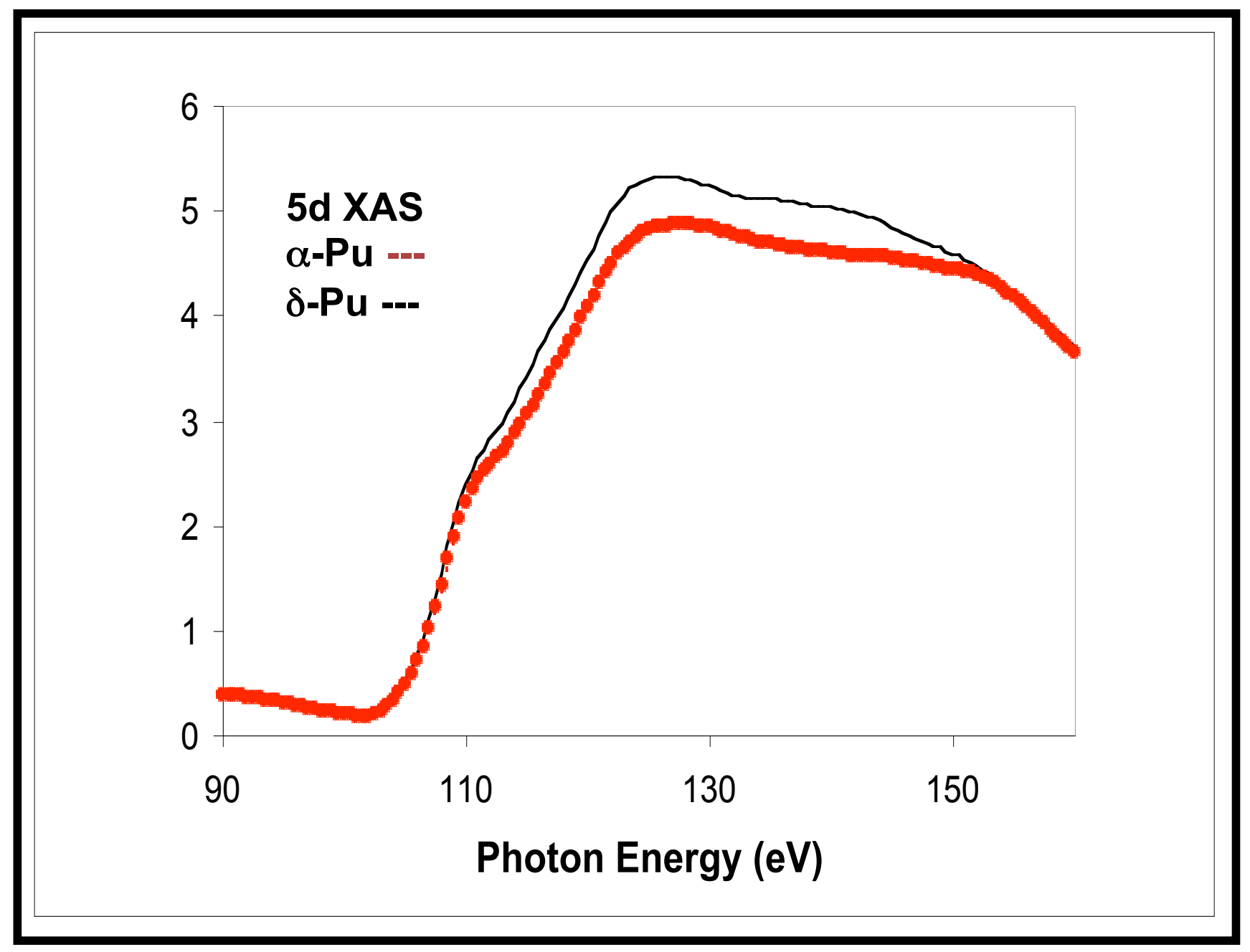

Figure 4

The XAS data for the $5 d$ to $5 f$ transition in Pu is shown here. The spectra are taken from Ref 16. 
On the electronic configuration in Pu: spectroscopy and theory

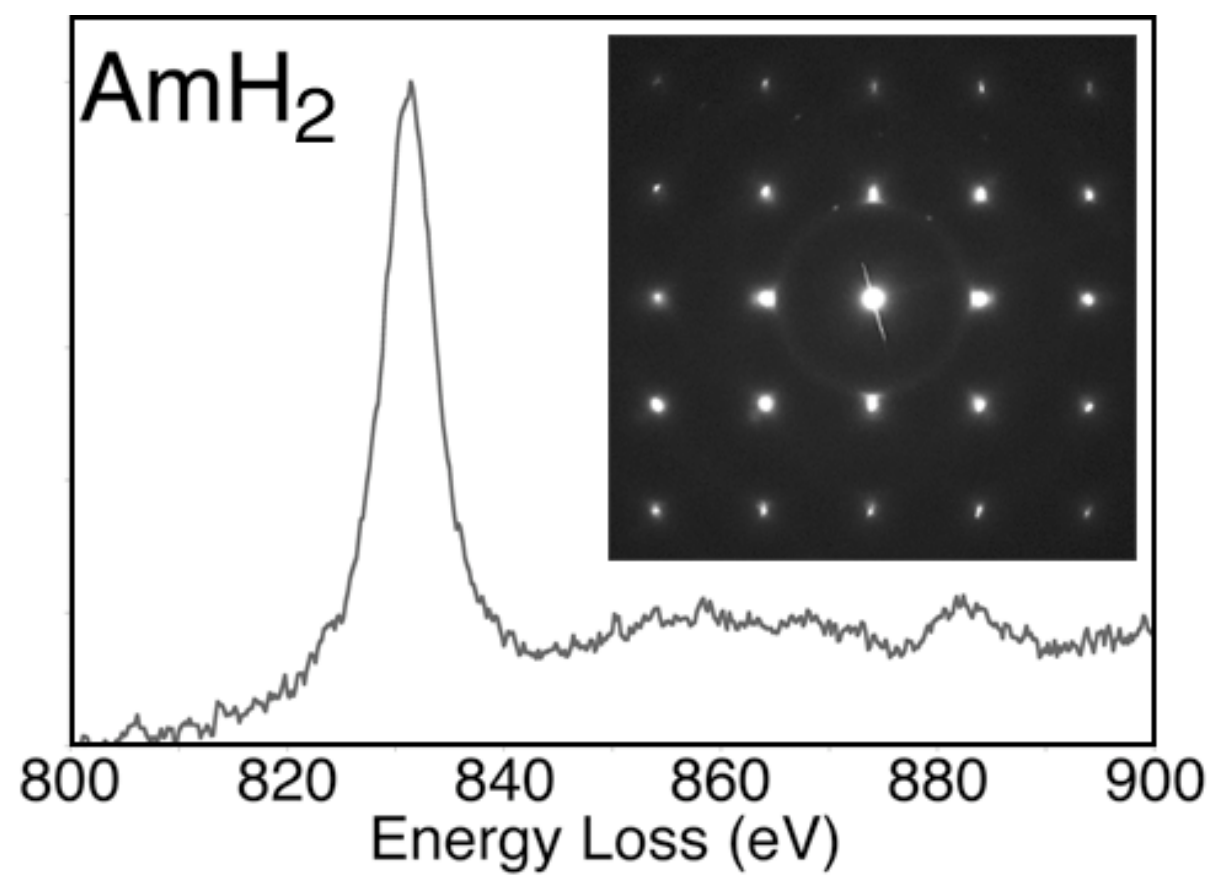

Figure 5

The electron diffraction and EELS data for the $4 d$ to $5 f$ transition of Am, from an $\mathrm{AmH}_{2} \mathrm{sample}$ is shown here. The data is taken from Ref 22. 


\section{On the electronic configuration in Pu: spectroscopy and theory}

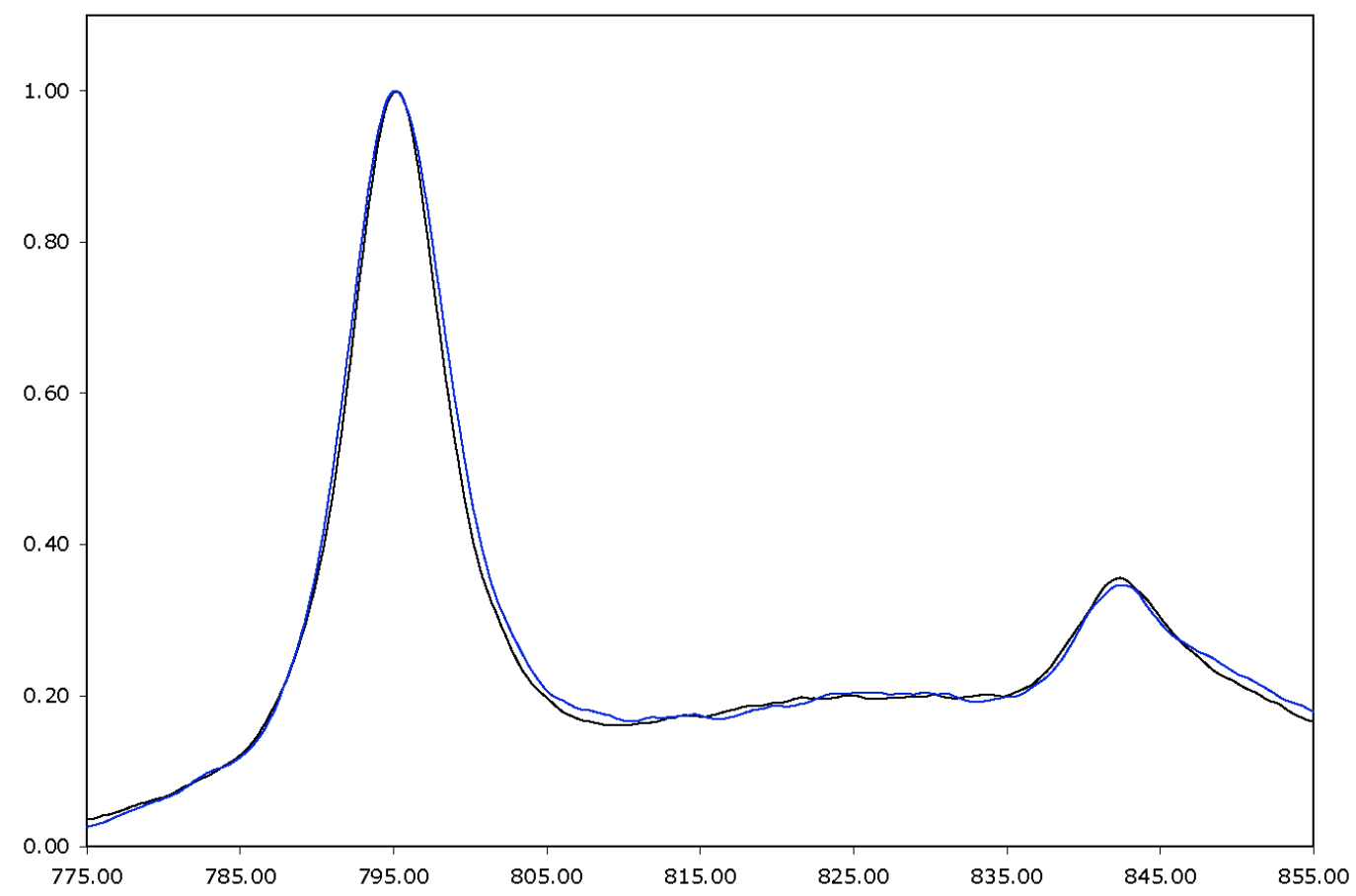

Figure 6

The EELS data for the $4 d$ to $5 f$ transition in $\square$ and $\square \mathrm{Pu}$ is shown here. The spectra are taken from Ref 22. 
On the electronic configuration in Pu: spectroscopy and theory

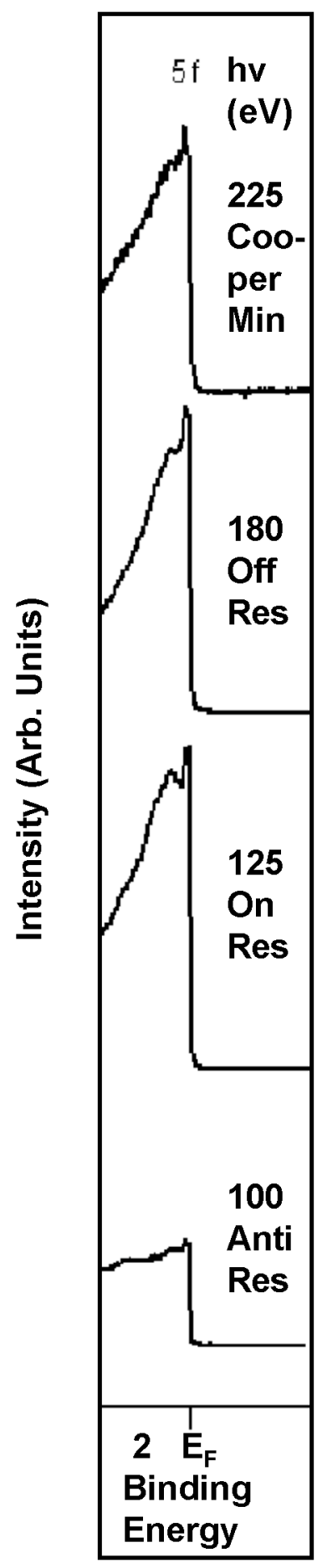

Figure 7

Shown here are photoelectron spectroscopy data for $\mathrm{Pu}$ at $\mathrm{h} \square=225,180,125$ and $100 \mathrm{eV}$. The data were taken from $\operatorname{Ref} 23$. 


\section{On the electronic configuration in Pu: spectroscopy and theory}
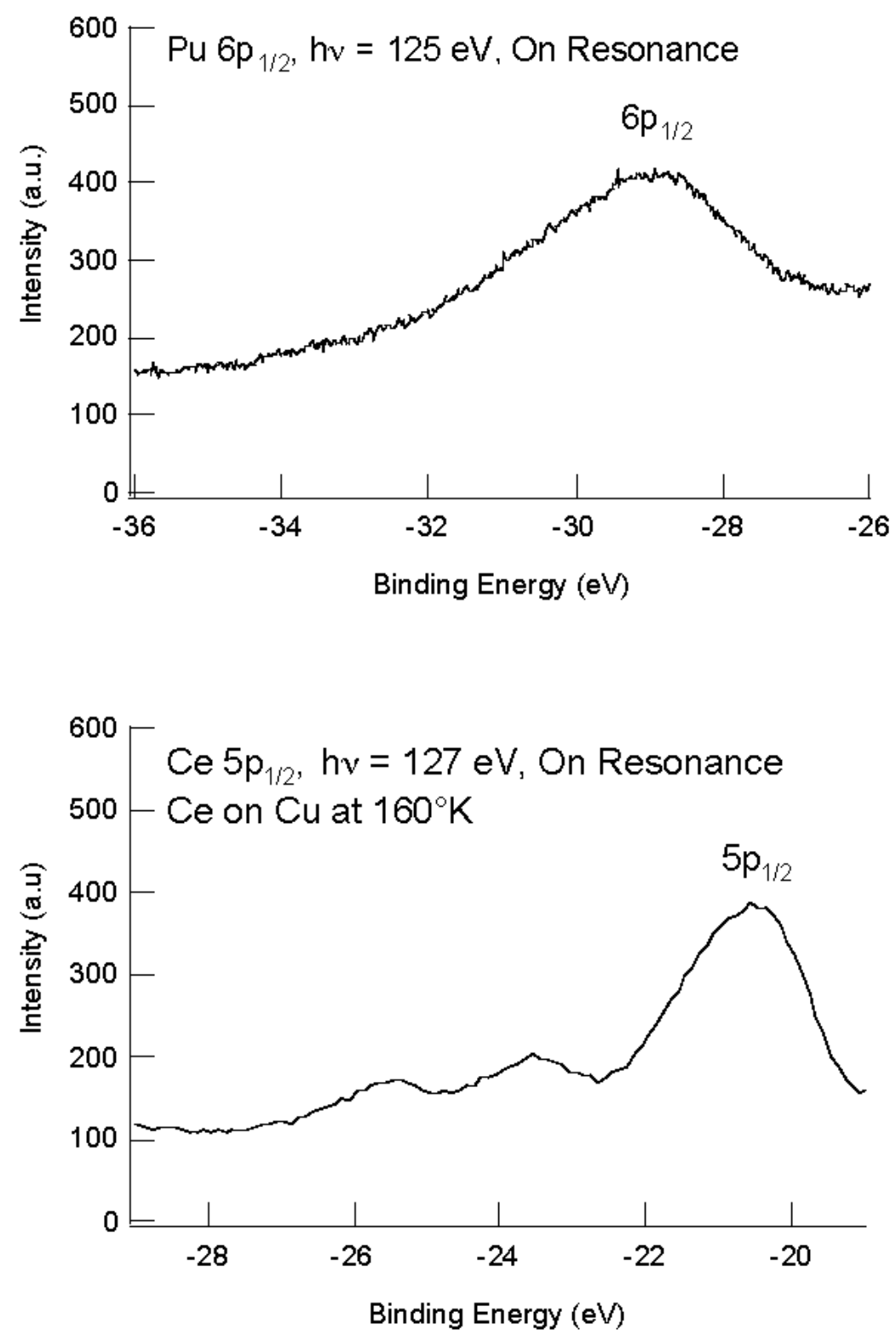

Figure 8

PES data of the Ce $5 p$ and $\mathrm{Pu} 6 p$ peaks are shown here. Both correspond to being on resonance, i.e., at the same energy as the $4 d$ to $4 f$ resonance in Ce and the $5 d$ to $5 f$ resonance in $\mathrm{Pu}$. See Ref 23 for more details. 


\section{On the electronic configuration in Pu: spectroscopy and theory}

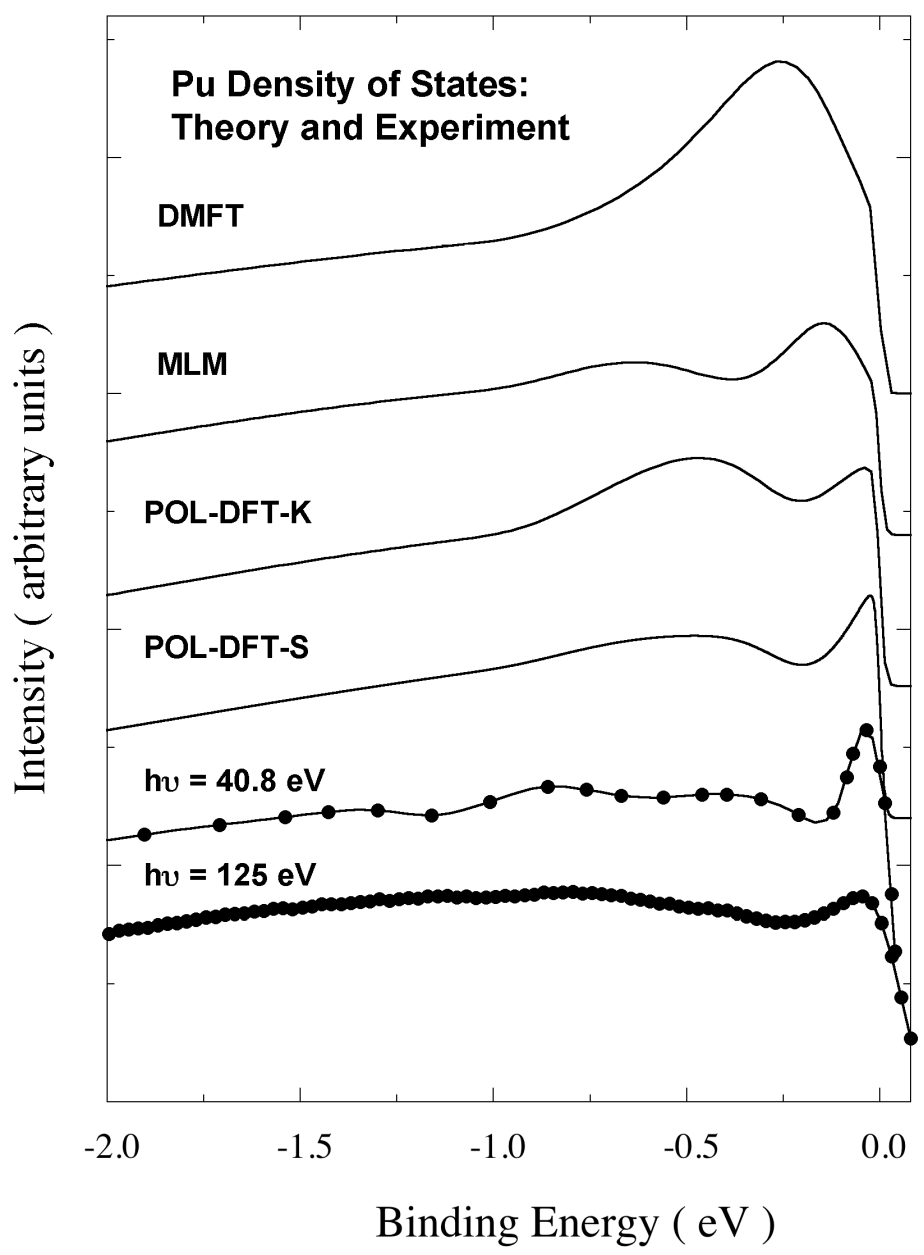

Figure 9.

Present $\square-\mathrm{Pu}$ photoemission spectra $(\mathrm{hv}=125 \mathrm{eV})$ and that of Arko et al. at hv $=40.8 \mathrm{eV}$ [10], compared with convoluted electronic density-of-states obtained from the mixed-level-model (MLM) [2], polarized DFT (DFT-POL-Söderlind et al and DFT-POL-Kutepov and Kutpova ) [6,32], and around-mean-field dynamical mean field theory (AMF-DMFT) [7]. Because of the increased instrumental broadening (about $01 . \mathrm{eV}$ ) of the $\mathrm{hv}=125 \mathrm{eV}$ data, the Fermi edge and the peak near the Fermi edge $(0.0 \mathrm{eV})$ are significantly broadened. Thus, while the synchrotron radiation results of Figure 7 validate the higher resolution data taken at hv $=40.8 \mathrm{eV}$, the best comparison with experiment must be made with the resonance lamp spectrum at hv $=40.8 \mathrm{eV}$. 
On the electronic configuration in Pu: spectroscopy and theory

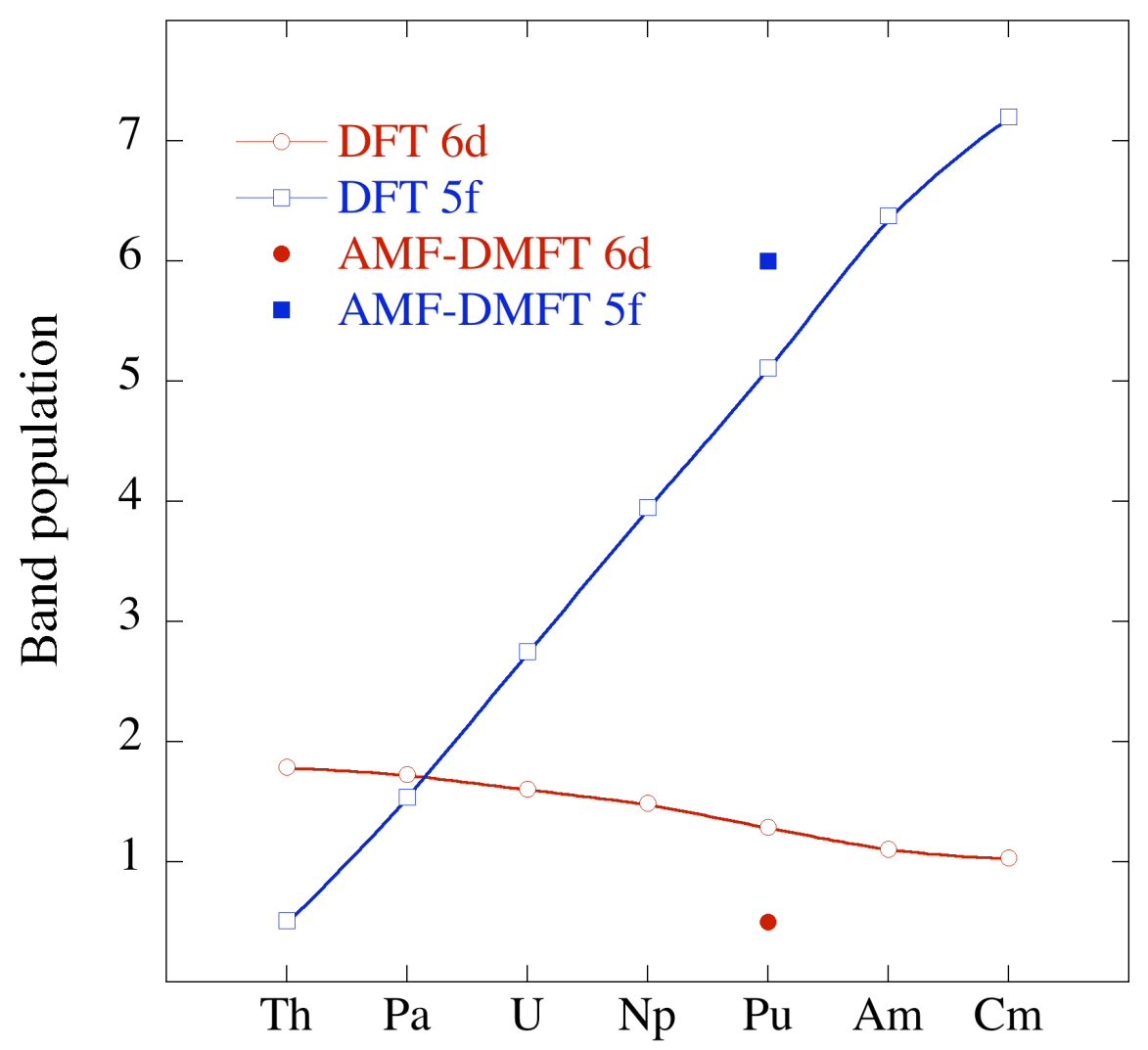

Figure 10. (Color online).

DFT and AMF-DMFT (Pu) $6 d$ and $5 f$ occupation numbers for Th-Cm. 


\section{On the electronic configuration in Pu: spectroscopy and theory}

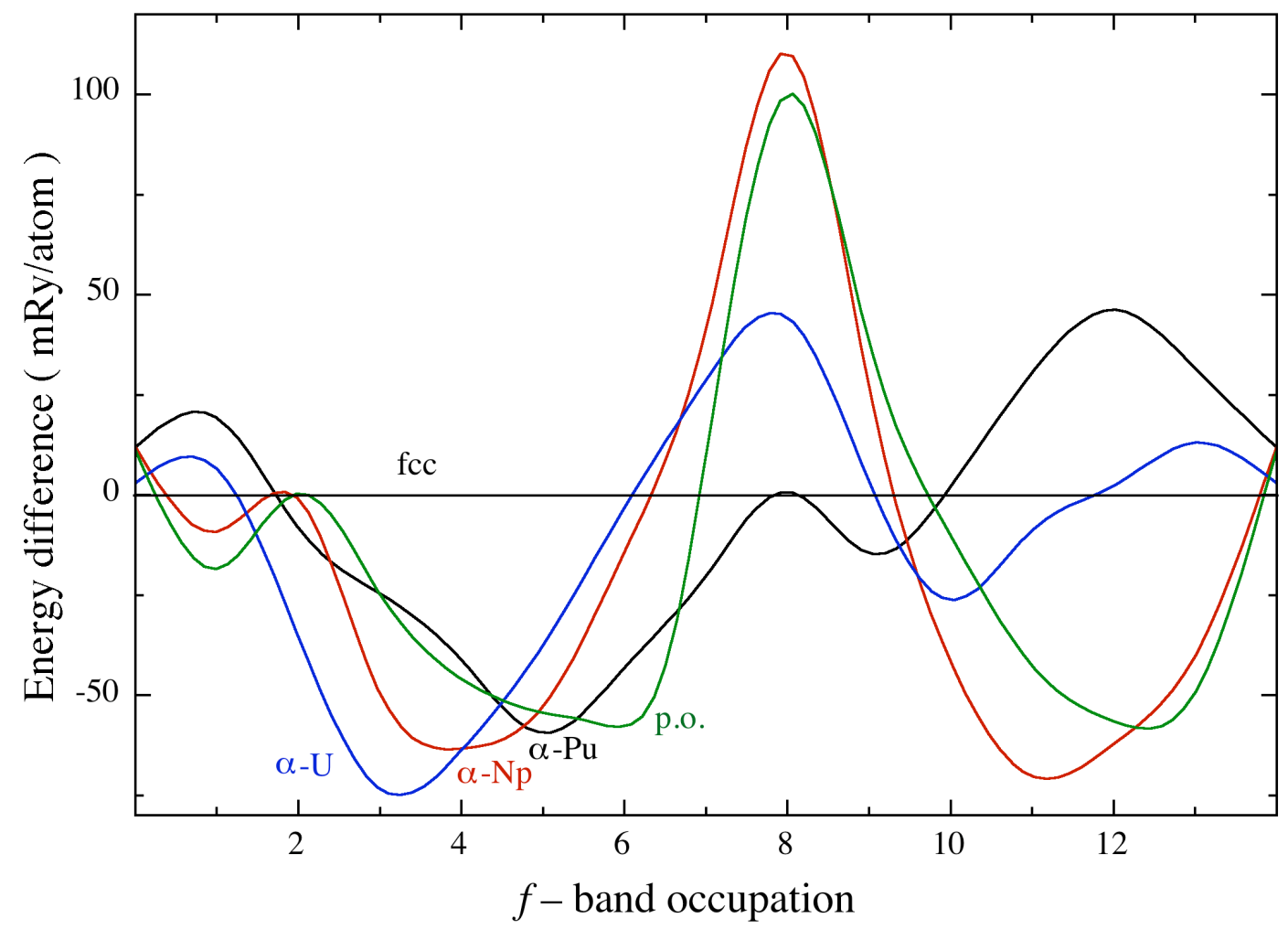

Figure 11. (Color online).

Energies for $\square-\mathrm{U}, \square-\mathrm{Np}, \square-\mathrm{Pu}$, and primitive orthorhombic (p.o.) geometries, relative to the fcc structure, as functions of $f$-band occupation, within a canonical band-energy model [42]. 